\title{
Infectious Diseases Research
}

National Cancer Institute

\section{Source}

National Cancer Institute. Infectious Diseases Research. NCI Thesaurus. Code C16003.

The study of infectious diseases in the laboratory 